\title{
The Role of Echocardiography in Diagnosing Space-Occupying Lesions of the Heart
}

\author{
Moluk Mirrasouli Ragland, DO and Tahir Tak, MD, PhD
}

\begin{abstract}
In contrast to primary cardiac tumors, which are less frequent and mostly benign in nature, the majority of intracardiac tumors are metastatic lesions. Cardiac ultrasound has evolved enormously since its emergence in the 1950s and is presently the modality of choice for imaging space-occupying lesions of the heart; it provides high quality, real-time images that are extremely valuable in the evaluation of cardiac masses. Although transthoracic echocardiography is an excellent initial diagnostic technique to evaluate and diagnose cardiac masses, transesophageal echocardiography provides superior image resolution and better visualization of cardiac masses in patients with suboptimal transthoracic echocardiography studies. Computed tomography and magnetic resonance imaging are additional tools used for cardiac imaging and may provide useful information in addition to that obtained by echocardiography, especially when the images obtained by the latter are suboptimal.
\end{abstract}

Keywords: Cardiac neoplasms; Diagnostic imaging; Echocardiography; Magnetic resonance; Imaging; Tomography

$\mathrm{T}_{\mathrm{r}, \mathrm{s}}$ he first effort to use pulse-reflected ultrasound to examine the heart was initiated by Dr. Helmut Hertz of Sweden in collaboration with Dr. Inge Edler. ${ }^{1}$ Despite their initial efforts at using ultrasound to examine the heart, neither Edler nor Hertz really anticipated that this technique would develop as rapidly as it did. ${ }^{1}$ In the 1950s, Dr. Effert and his colleagues in Germany began to duplicate Edler's work describing mitral stenosis. During their work, Effert and his group detected left atrial masses. Since the initial ultrasonographic demonstration of an atrial myxoma by Effert and Domanig in 1959, the value of echocardiography for diagnosing cardiac mass lesions has become well established. ${ }^{2}$ Numerous advances have occurred in the last five decades. The evolution of echocardiography involves the development of its many modalities of A-mode, M-mode, contrast echocardiography, two-dimensional echocardiography (2DE), Doppler ultrasound, transesophageal echocardiography (TEE) and intravascular applications. ${ }^{1}$ In this review, we describe various cardiac masses and the role of 2DE in the evaluation of these space-occupying lesions. A discussion and review of other imaging modalities, such as computed tomography (CT) and magnetic resonance imaging (MRI), are also presented.

\section{Structures Misinterpreted for Cardiac Masses}

Abnormal masses (tumors, thrombi, vegetations) must be distinguished from normal cardiac structures that may mimic a mass. The echocardiographic evaluation of intracardiac masses is dependent on the ability to distinguish normal from abnormal findings. ${ }^{1}$ Erroneous diagnoses may lead to inappropriate medical therapy and even inappropriate surgery. ${ }^{3}$ Recognition of such normal variants depends on image quality and technique, as well as a physician's experience. ${ }^{1}$ There are several normal structures and variants that may mimic a cardiac mass. The Eustachian valve, Chiari network, crista terminalis, pectinate muscles, moderator band, trabeculations, interatrial septal aneurysm and lipomatous hypertrophy of the interatrial septum are some examples of normal structures that are frequently mistaken for pathologic entities (figure 1). 4,5 Other entities which may cause confusion and appear prominently on echocardiographic images include surgical sutures, internal pacemaker electrodes and intracardiac catheters. Table 1 summarizes these commonly encountered normal structures by their location in various cardiac chambers.

Ultrasound artifacts are common, even in high-quality studies, and may be mistaken for pathologic conditions. Therefore, one
Reprint Requests: Tahir Tak, MD, PhD, Department of Medicine, University of North Texas Health Science Center, 855 Montgomery Road, $4^{\text {th }}$ Floor, Fort Worth, Texas 76107, Tel: 817-735-2332; Fax: 817-735-2673, E-mail: ttak@hsc.unt.edu
Received: August 18, 2005

Revised: November 30, 2005

Accepted: December 13, 2005 

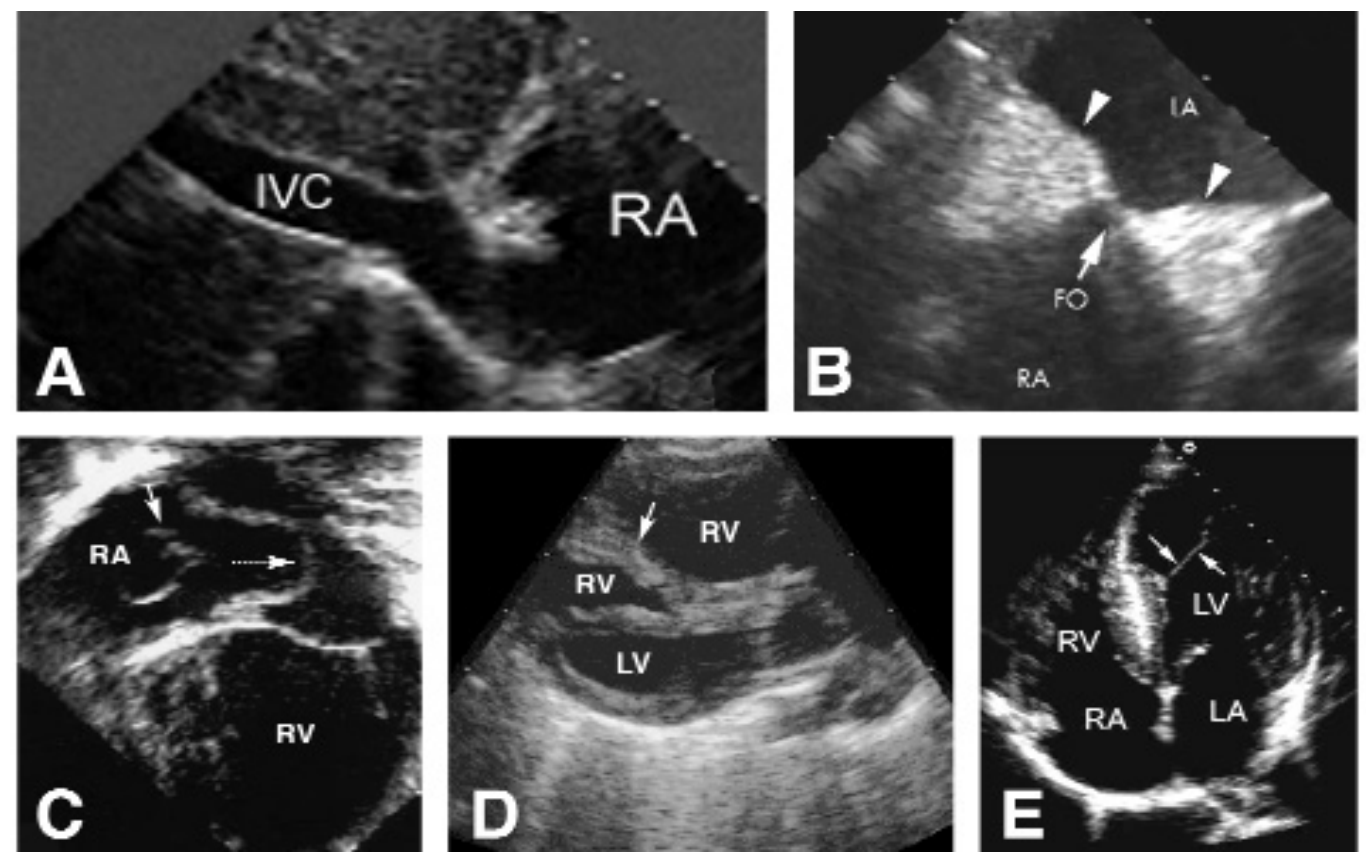

Figure 1. (A) The Eustachian valve is demonstrated extending from the inferior vena cava to the right atrium. [Reproduced with permission from E-chocardiography Journal: An Electronic Journal of Cardiac Ultrasound (http://www2.umdnj.edu/ shindler/anatomy.html). Copyright 1995-2006 Daniel Shindler, MD.] (B) Transoesophageal echocardiogram showing the classic "dumbbell" shaped lipomatous hypertrophy of the intra-atrial septum (arrow heads). Note that the bright echogenicity of thickened tissue clearly spares the fossa ovalis (arrow). FO, fossa ovalis; LA, left atrium; RA, right atrium. [Reproduced with permission of the BMJ Publishing Group from Nadra et al. Lipomatous hypertrophy of the interatrial septum: a commonly misdiagnosed mass often leading to unnecessary cardiac surgery. Heart 2004;90:e66. Copyright 2004 BMJ Publishing Group. All rights reserved.] (C) Two-dimensional transthoracic echocardiography showing the aneurysmal atrial septum (dotted arrow) and the Chiari network (solid arrow) in the right atrium. RA, right atrium; RV, right ventricle. [Reproduced with permission of the Texas Heart Institute from McMahon et al. Echocardiographic features of Chiari's Network in association with tricuspid atresia. Tex Heart Inst J 2000;27:312-313. Copyright 2000 Texas Heart Institute. All rights reserved.] (D) This echocardiogram demonstrates a prominent muscle bundle, called the moderator band (arrow), in the right ventricle that extends from the apical lateral wall to the distal interventricular septum. [Photo from The Encyclopedia of Congenital Heart Disease (http://www.kumc.edu/kumcpeds/cardiology/allechos.html). Reproduced courtesy of Scientific Software Solutions, Inc.] (E) This four-chamber view demonstrates a false tendon (arrows) in the left ventricular apex. LA, left atrium; RA, right atrium; RV, right ventricle. [Reproduced with permission of Lippincott Williams \& Wilkins from Feigenbaum et al. Feigenbaum's Echocardiography. 6 th ed. Philadelphia, PA: Lippincott Williams \& Wilkins; 2005. 702 (Figure 21.1).]

has to exercise caution in interpreting these artifacts. Near-field clutter and reverberations are examples of artifacts often confused with pathology (e.g., apical thrombi) on 2DE. ${ }^{1}$ Proper transducer selection and the use of multiple acoustic windows are among the strategies that can be used to avoid potential misinterpretations. ${ }^{1}$

In addition to normal variants and echocardiographic artifacts, extracardiac masses may compress the heart and create a mass effect. Examples of such masses (pseudotumors) include mediastinal tumors, coronary aneurysms or hiatal hernias. ${ }^{1}$ An example of a hiatal hernia that shows a herniated portion of the stomach appearing as a mass within the atrium is shown in figure 2. The diagnosis of hiatal hernia can be verified by having the patient drink a carbonated beverage during the transthoracic echocardiographic (TTE) examination. ${ }^{1}$

\section{Primary Cardiac Tumors}

With an incidence of approximately $0.02 \%$ in autopsy series, primary tumors of the heart are far less common than metastatic tumors of the heart. ${ }^{5}$ About $75 \%$ of all primary cardiac tumors are benign and primary malignant tumors account for the other
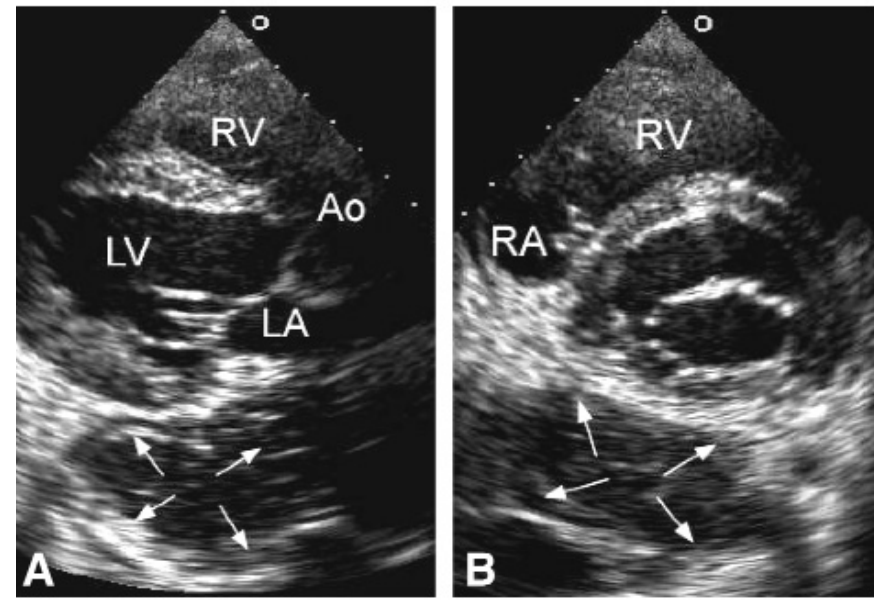

Figure 2. Echocardiogram demonstrating "hiatal hernia" as an echo-free space (arrows) behind the heart. (A) Parasternal long-axis view. (B) Parasternal short-axis view. LA, left atrium; $\mathrm{RA}$, right atrium; Ao, aorta; LV, left ventricle; RV, right ventricle. 
Table 1. Normal variants and benign conditions often misinterpreted as pathologic.

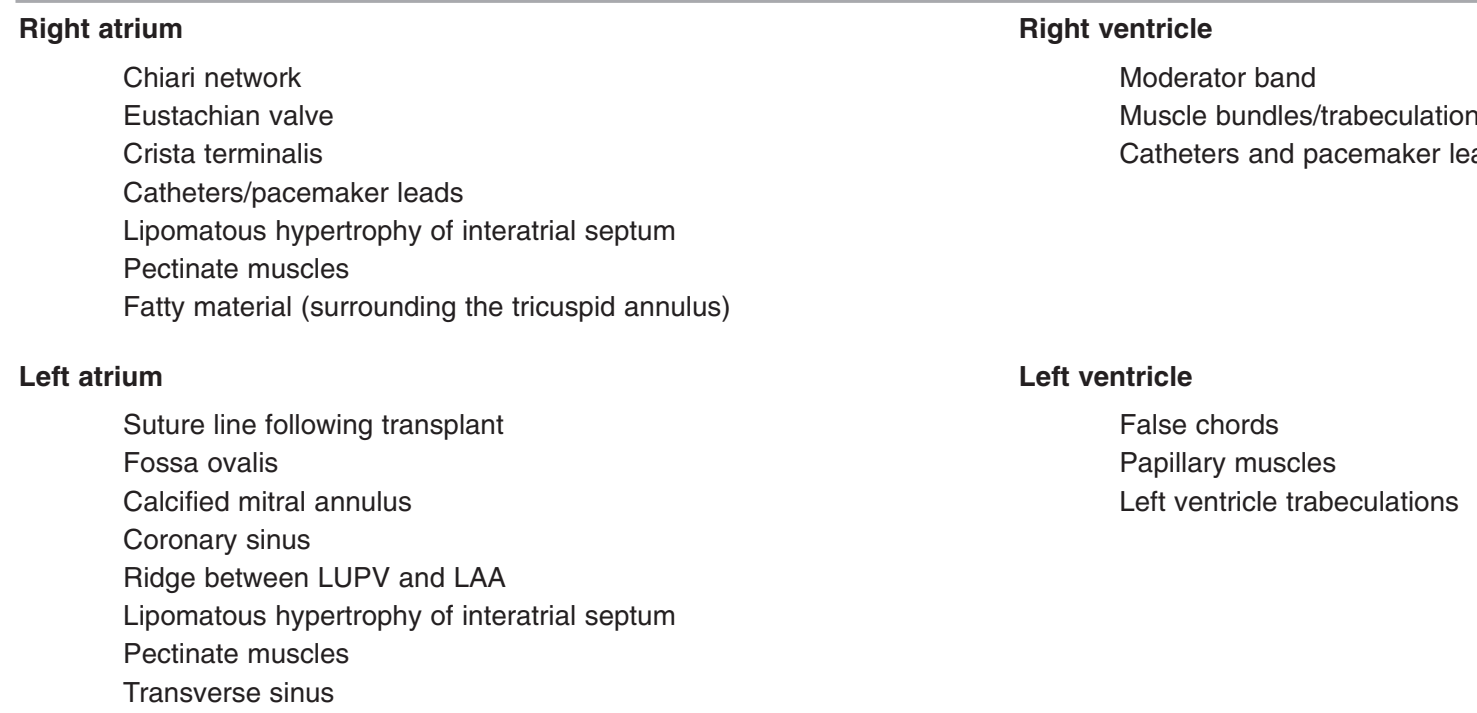

Aorta

Brachiocephalic vein

Innominate vein

Pleural effusion

LAA, left atrial appendages; LUPV, left upper pulmonary vein.

Reproduced with permission of Lippincott Williams \& Wilkins from Feigenbaum et al. Feigenbaum's echocardiography. $6^{\text {th }}$ ed. Philadelphia, PA: Lippincott Williams \& Wilkins; 2005. 701 (Table 21.1).

$25 \% .{ }^{6,7}$ Common primary cardiac tumors are listed in table 2 in order of frequency.

\section{Benign Cardiac Tumors}

Myxoma

Myxomas are the most common benign cardiac tumors, accounting for $30 \%$ of all primary cardiac tumors. They occur most commonly in the left atrium (75\%), but can arise in the right atrium $(23 \%)$ or the ventricles $(2 \%) .{ }^{6}$ Cardiac myxomas usually arise from fossa ovalis of the interatrial septum and protrude into the atrium (figure 3A). They may intermittently cause obstruction to left ventricular filling, especially when they are large in size and in the left atrium. This typically causes a "plop" on auscultation. ${ }^{8}$ Patients with myxoma present with a triad of embolization, intracardiac obstruction and constitutional signs. ${ }^{9}$ Constitutional symptoms include arthralgias, rash, fever, weight loss and fatigue..$^{5}$ These constitutional symptoms are due to release of vasoactive products from the tumor or an autoimmune response invoked by tumor products or production of cytokine interleukin-6 (IL-6) by cardiac myxomas. ${ }^{10}$

The gross appearance of cardiac myxoma is variable. ${ }^{11}$ However, they are generally polypoid, mostly pedunculated, round or oval with a smooth surface and often covered with thrombi. ${ }^{12}$ Due to the friability of the left atrial myxoma, systemic emboli are estimated to occur in approximately $40 \%$ to $50 \%$ of the cases. ${ }^{10}$ The most frequent site of myxomatous emboli is the cerebral vessels, including the retinal arteries. ${ }^{10}$ The extremities are the next most common sites of embolic phenomena of atrial myxoma. Cutaneous findings include telangiectasia, livedo reticularis, tender, blanching or nonblanching erythematous macule or papule, and serpiginous or ulcerating lesions on extremities. ${ }^{10}$ Knowledge of the various cutaneous findings associated with myxomas may help in the early recognition of these cardiac masses. ${ }^{10}$

Two-dimensional echocardiography is the imaging modality of choice, classically revealing a mobile tumor connected to the interatrial septum by a narrow stalk. ${ }^{5}$ Myxomas usually have heterogeneous echogenecity and occasional calcifications. The most important clue to the diagnosis is their location in the left atrium and origin from the mid-portion of the atrial septum. ${ }^{1}$ It is, therefore, important to identify the site of tumor attachment during the echocardiographic examination. This allows us to differentiate it from other masses, e.g., a thrombus. Given a typical presentation, echocardiography is virtually diagnostic of myxoma. ${ }^{1}$ TTE is usually sufficient to make the diagnosis, but if the results are suboptimal, TEE should be employed. ${ }^{5}$ Investigations have shown that MRIs and CT scans have no incremental value in diagnosing cardiac myxoma when the echocardiography is of good quality and identifies the tumor and its site of attachment. The thin, delicate stalk of myxoma usually cannot be defined at CT or MR imaging. ${ }^{11}$ Although a generally narrow base of attachment can be seen on CT and MRI, it is suggestive of, but not definitive for, a diagnosis of myxoma. ${ }^{11}$ 
Table 2. Relative frequency of primary cardiac tumors.

\begin{tabular}{lc}
\hline Type & Percent \\
\hline Benign & \\
Myxoma & 30 \\
Lipoma & 10 \\
Papillary fibroelastoma & 8 \\
Rhabdomyoma & 6 \\
Fibroma & 3 \\
Hemangioma & 2 \\
Teratoma & 1 \\
& \\
Malignant & \\
Angiosarcoma & 8 \\
Rhabdomyosarcoma & 5 \\
Fibrosarcoma & 3 \\
Mesothelioma & 3 \\
Lymphoma & 2 \\
Leiomyosarcoma & 1 \\
\hline
\end{tabular}

Reproduced with permission of Lippincott Williams \& Wilkins from Feigenbaum et al. Feigenbaum's echocardiography. $6^{\text {th }}$ ed. Philadelphia, PA: Lippincott Williams \& Wilkins; 2005. 703 (Table 21.3).
Familial myxomas constitute $10 \%$ or less of all myxomas..$^{5}$ In contrast to sporadic myxomas, which typically present at an older age, patients with familial myxoma tend to present earlier (median age, 20 years) and are more likely to have myxomas in atypical locations, to have multiple tumors, are more likely to develop recurrent tumors, and have associated dermatological and endocrine abnormalities. ${ }^{13}$ Vidaillet et al. ${ }^{13}$ described these distinctive clinical features of familial myxoma as "syndrome myxoma," which is also known as "Carney complex" of myxomas, spotty skin pigmentation and endocrine overactivity. A careful search for multiple cardiac tumors should be made to differentiate sporadic from familial myxomas. Due to the autosomal dominant transmission of Carney complexes, identification of multiple cardiac myxomas in a young individual should prompt echocardiographic screening of first-degree relatives. ${ }^{14}$ Postoperatively, these patients should be observed closely for recurrence of myxomas due to a recurrence rate of $12 \%$ to $22 \% .^{5}$

\section{Lipomas}

Lipomas are the second most common benign cardiac tumors (with a frequency of $10 \%$ of benign cardiac tumors) ${ }^{1}$ and are usually seen in the left ventricle, right atrium and interatrial
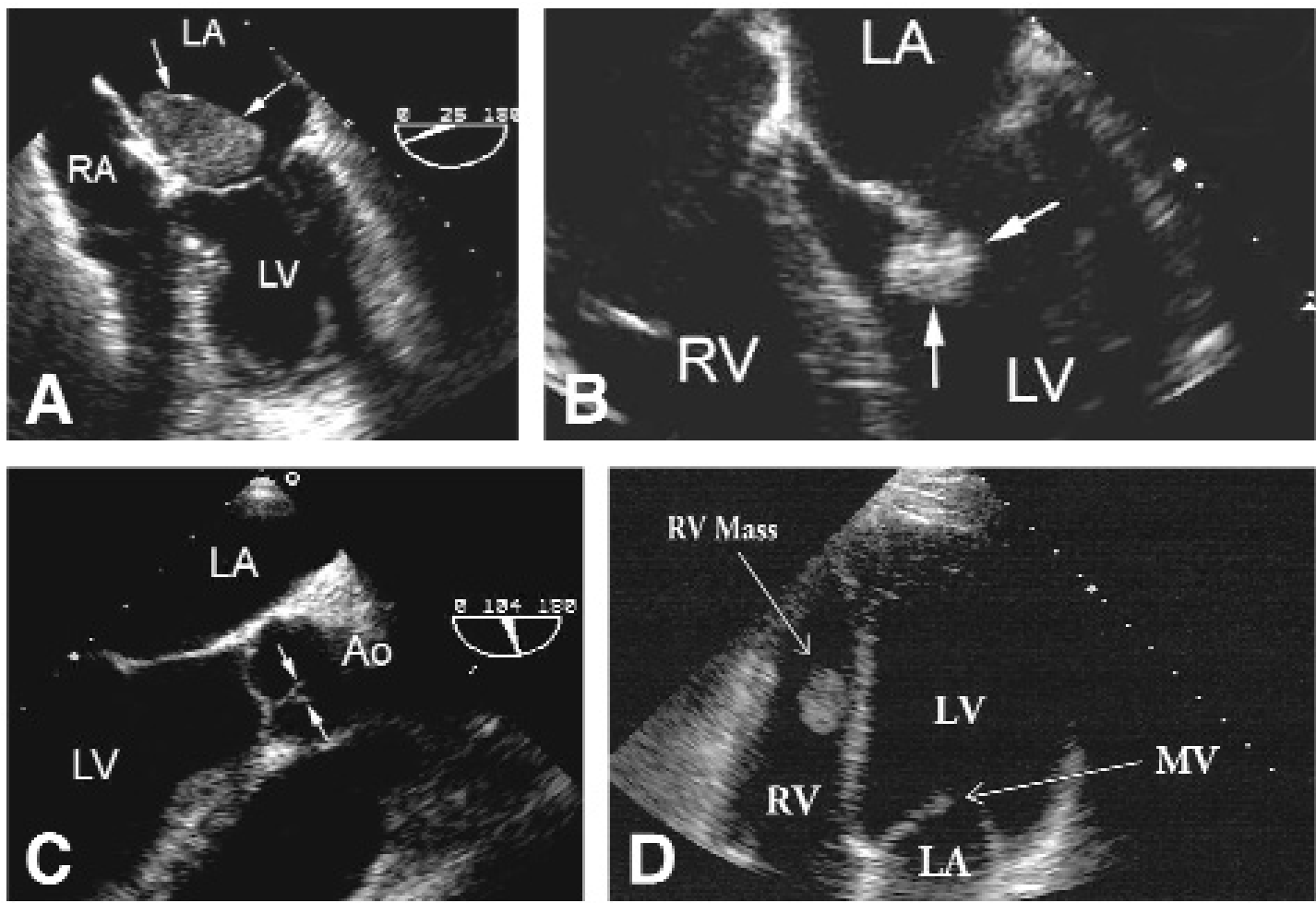

Figure 3. (A) This transesophageal imaging shows a myxoma (arrows) in the left atrium that is attached to the fossa ovalis. (B) A papillary fibroelastoma (arrows) of the mitral valve is demonstrated. The tumor was attached by a small pedicle to the anterior leaflet and was highly mobile. AV, aortic valve; LA, left atrium; LV, left ventricle; RV, right ventricle. (C) An example of Lambl excrescence of the aortic valve is demonstrated (arrows). Ao, aorta; LA, left atrium; LV, left ventricle. (D) On apical four-chamber echocardiographic view, a right ventricular mass is noted to be attached to the interventricular septum. LA, left atrium; LV, left ventricle; MV, mitral valve; RV, right ventricle; RV mass, right ventricular mass. [Panels A-C: Reproduced with permission of Lippincott Williams \& Wilkins from Feigenbaum et al. Feigenbaum's Echocardiography. 6th ed. Philadelphia, PA: Lippincott Williams \& Wilkins; 2005. 703 (Figure 21.3 A), 704 (Figure 21.6 A), 706 (Figure 21.9). Panel D: Reproduced with permission of American College of Chest Physicians from Krasuski et al. Cardiac rhabdomyoma in an adult patient presenting with ventricular arrhythmia. Chest 2000;118:1217-1221. Copyright 2000 American College of Chest Physicians. All rights reserved.] 
septum. ${ }^{5}$ About $50 \%$ arise subendocardially, 25\% subepicardially and $25 \%$ from the myocardium. ${ }^{12}$ Their size can range from $1 \mathrm{~cm}$ to $15 \mathrm{~cm}$.

Echocardiography typically reveals a homogenenous, hyperechoic mass, but these findings are not diagnostic. ${ }^{5}$ The echocardiographic appearance of lipomas can vary depending on their location. Lipomas in the pericardial space are usually hypoechoic, whereas intracavitary lipomas are homogenous and hyperechoic. ${ }^{11}$ The etiology for these findings is not known. Since both CT and MRI can help identify fat with a high degree of specificity, they can be used to diagnose cardiac lipomas without equivocation. ${ }^{11}$ For this reason, reports of cardiac lipomas over the past 10 years to 15 years have tended to emphasize the role of CT and MRI. ${ }^{11}$ Studies have shown that the best imaging modality is MRI. ${ }^{5}$ On MRI, lipomas have homogeneous increased signal intensity on T1-weighted images that decrease with fat-saturated sequences. ${ }^{11}$

Lipomatous hypertrophy (mentioned earlier under normal variants) is often discussed with cardiac lipomas, but it does not constitute a true neoplasm. It is thought to be benign and rarely associated with clinical manifestations. ${ }^{1}$ In contrast to true cardiac lipomas that can occur at any age, lipomatous hypertrophy of the interatrial septum is seen in obese and relatively older patients. ${ }^{11}$ Lipomatous hypertrophy has a reported incidence of $1 \%$ to $8 \%$ and is usually an incidental finding because most patients with this condition are asymptomatic. ${ }^{4}$ Patients with lipomatous hypertrophy are generally obese and may be difficult to image with conventional TTE. ${ }^{15}$ TEE can provide high quality imaging in these patients and shows the classic dumbbell or hourglass appearance of septum caused by fatty infiltration of the proximal and distal portions of the septum with sparing of the fossa ovalis area (figure 1B). ${ }^{4}$

\section{Papillary fibroelastoma}

Papillary fibroelastomas are small tumors $(\leq 1 \mathrm{~cm})$ that account for approximately $8 \%$ of primary benign cardiac tumors. ${ }^{1}$ They are usually found in older patients with the average age at detection of 60 years. ${ }^{5}$ They commonly arise on the mitral and, less commonly, on the aortic valve, but can also be found on other endocardial surfaces (figure 3B). Due to their small size, generally $0.5 \mathrm{~cm}$ to $2.0 \mathrm{~cm}$ in diameter, and their occurrence on the valves, they may be difficult to distinguish from valvular vegetations. However, unlike vegetations, fibroelastomas are more often found on the downstream side of the valve, and despite their valvular attachment, valvular dysfunction is uncommon. ${ }^{5}$ If valvular dysfunction (valvular destruction or regurgitation) is present, valvular vegetations rather than papillary fibroelastoma should be suspected. A characteristic shimmer or vibration at the tumor-blood interface, ascribed to the finger-like projections of the tumor, has been described and is best appreciated on TEE. ${ }^{5}$ There is some confusion as to whether fibroelastomas are distinctly different from Lambl's excrescences, which are smaller in size and seen more frequently in elderly patients. ${ }^{1}$ Lambl's excrescences are usually along the closure margins of the valve cusps (figure 3C).
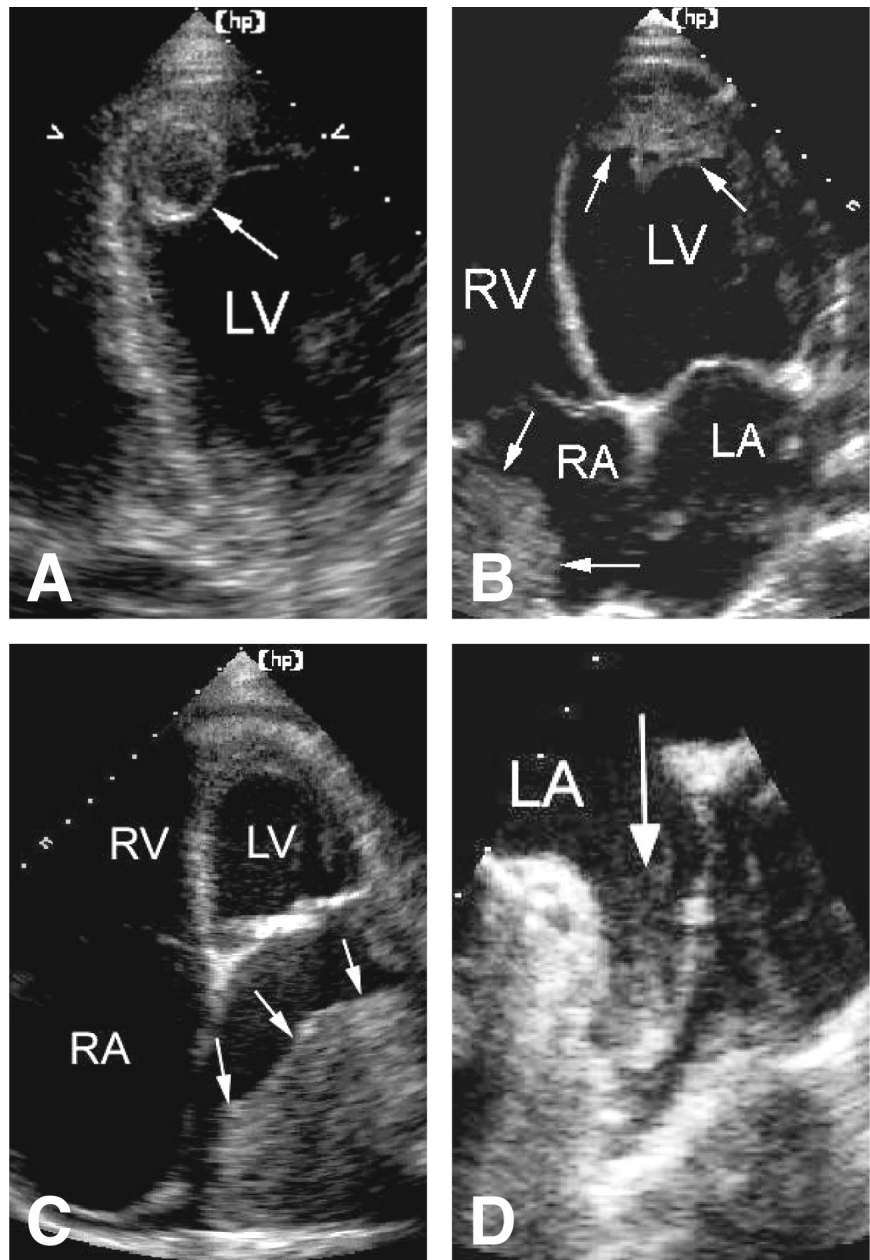

Figure 4. (A) A large, circular mass (arrow) consistent with a thrombus is demonstrated in the left ventricle. (B) Multiple thrombi are noted in a patient with severe heart failure due to dilated cardiomyopathy. A left ventricular apical thrombus and a large right atrial thrombus are indicated by the arrows. LA, left atrium; LV, left ventricle; $R A$, right atrium; $R V$, right ventricle. $(C) A$ very large left atrial thrombus (arrows) is demonstrated. RA, right atrium; LV, left ventricle; $R V$, right ventricle. (D) A small thrombus within the left atrial appendage is demonstrated. LA, left atrium. [Reproduced with permission of Lippincott Williams \& Wilkins from Feigenbaum et al. Feigenbaum's Echocardiography. $6^{\text {th }}$ ed. Philadelphia, PA: Lippincott Williams \& Wilkins; 2005. 716 (Figure 21.28 C), 717 (Figure 21.30 A), 719 (Figure 21.34), 721 (Figure 21.39).]

Papillary fibroelastomas are friable tumors that are likely to form surface thrombus and have the potential to embolize. ${ }^{16,17}$ Anticoagulation does not appear to protect against these embolic events. ${ }^{5}$ Surgical treatment (especially of left-sided lesions) has been described in cases where there has been recurrence of strokes, presumably secondary to embolism. ${ }^{5}$

\section{Rhabdomyoma}

Rhabdomyomas have a frequency of approximately $6 \%$ of all benign tumors and commonly occur in infants and children. ${ }^{1}$ Approximately three-fourths occur in patients younger than 1 year and are extremely uncommon in the adult patient. 5,18 They occur either within a cavity, sometimes as a 
pedunculated mass, or embedded within the myocardium (figure 3D). Such tumors can grow quite large and obstruct blood flow within the heart. ${ }^{1}$ They occur with equal frequency in the right and left ventricular and septal myocardium, can involve either one or both atria, and nearly all are multiple. ${ }^{5}$ The type and severity of symptoms depend on the location and size of the tumors. Heart block is the most common manifestation, but paroxysmal supraventricular tachycardia, ventricular tachycardia and sudden cardiac death have also been reported. ${ }^{5}$ Intracavitary tumors may lead to signs and symptoms of congestive heart failure secondary to obstruction of blood flow. 5 Rhabdomyomas are strongly associated with tuberous sclerosis, which is an autosomal dominant syndrome characterized by hamartomas in several organs, epilepsy, mental deficiency and adenoma sebaceum. Several studies show that at least $80 \%$ of patients with cardiac rhabdomyomas have tuberous sclerosis. ${ }^{5}$ However, cardiac rhabdomyomas in tuberous sclerosis patients is often present without major arrhythmia or hemodynamic obstruction. ${ }^{19}$ Before the advent of echocardiography, the majority of published reports about cardiac rhabdomyoma were based on autopsy studies. ${ }^{19}$ Echocardiography is usually adequate for diagnosis and typically reveals multiple small, lobulated, homogeneous, hyperechoic intramural tumors. ${ }^{5}$ Prognosis of asymptomatic patients is usually excellent, however, children with symptomatic cardiac rhabdomyoma have a poor prognosis. It has been reported that $78 \%$ of children with symptomatic rhabdomyoma died before the age of 1 year. ${ }^{19}$ Fortunately, $50 \%$ or more of rhabdomyomas regress spontaneously after infancy and even resolve completely without the need for intervention. ${ }^{18}$ Thus, in the absence of symptoms, surgery is not indicated. In a study that aimed to investigate the mechanism of spontaneous tumor regression, it was concluded that the cytoplasmic content in cardiac rhabdomyoma were degraded via ubiquitin pathway and the rate of cell death was increased in cardiac rhabdomyoma. ${ }^{20}$ If rhabdomyomas are confirmed with biopsy, the patient should be followed up with periodic echocardiograms rather than surgery. ${ }^{5}$ Surgery is reserved for symptomatic patients with significant hemodynamic obstruction. ${ }^{19}$ Even if surgery is indicated, complete removal of cardiac rhabdomyomas is not always possible because these tumors are usually located deep in the myocardium. However, limited surgical excision is often effective because the residual tumor usually involutes. ${ }^{19}$

\section{Fibromas}

Fibromas are uncommon benign tumors that are most often seen in children (second most common benign pediatric cardiac tumor) but are rare in adults. 3,21 They are usually found in the ventricular myocardium and usually occur within the left ventricular free wall, anterior free wall or the interventricular septum. ${ }^{21}$ They vary in size from $3 \mathrm{~cm}$ to 10 $\mathrm{cm}$ and have central calcifications. On echocardiography, they appear as distinct, highly echogenic and well-demarcated masses that often extend into the cavity of the ventricle. ${ }^{1}$ They may be confused with rhabdomyomas. Although benign, they occasionally result in obstruction to left ventricular filling and have been associated with ventricular arrhythmias. ${ }^{1}$ Like lipomas, they are not associated with systemic embolization.
There is an increased prevalence of cardiac fibromas in Gorlin syndrome, also known as nevoid basal cell carcinoma syndrome. The incidence of this autosomal dominant syndrome is uncommon, but not rare and it is marked by different signs at different ages, making recognition difficult. ${ }^{21}$ It is associated with basal cell carcinoma, odontogenic keratocysts of the mandible, skeletal anomalies and a tendency toward neoplastic growth in several organ systems. ${ }^{11}$ Gorlin syndrome is characterized by skeletal (spine and rib) abnormalities, calcification of the falx cerebri (strongly suggestive of this syndrome), multiple basal cell carcinomas (the hallmark of this syndrome), small palmar or plantar pits due to superficial keratin defects and multiple jaw keratocysts (often the initial sign of the syndrome). ${ }^{21}$ Both cardiac and ovarian fibromas have been associated with this syndrome. ${ }^{22}$ Cotton et al ${ }^{21}$ describe a case report of a 2-year-old child with a cardiac fibroma that was subsequently diagnosed as having Gorlin syndrome. Based on these findings, it is recommended that patients with a nevoid basal cell carcinoma be evaluated for cardiac fibromas. Echocardiography typically reveals an intramural, homogeneous, echogenic mass. ${ }^{5}$ Approximately $70 \%$ of fibromas are symptomatic, causing mechanical interference with intracardiac flow, ventricular systolic function or conduction disturbances. Sudden cardiac death occurs in $14 \%$ of patients with fibromas, typically in infants. ${ }^{5}$ Cardiac fibromas are often associated with arrhythmias and are the second most common primary cardiac tumor associated with sudden death after endodermal heterotopia of the atrioventricular node. ${ }^{11}$ Given the risk of fatal arrhythmias, resection is usually recommended even in asymptomatic cases. Other rare benign tumors include teratoma (which occurs mostly in infants), hemangioma and mesothelioma. ${ }^{3}$

\section{Malignant Cardiac Tumors}

A quarter of all cardiac tumors are malignant, the majority of which are angiosarcomas or rhabdomyosarcomas. ${ }^{8}$ Malignant primary cardiac tumors are rare and nearly all $(95 \%)$ of these are sarcomas. Sarcomas are common between the third and fifth decades of life and most frequently affect the right atrium. ${ }^{8}$ Lymphomas account for the remaining $5 \%$ of primary malignant cardiac tumors. ${ }^{5}$ Malignant tumors usually begin as intramural and expand rapidly extending to the pericardium and/or cardiac chambers. Primary cardiac malignancies present a clinical dilemma and are often asymptomatic until they become large, and even then, they produce nonspecific symptoms. ${ }^{23}$ Malignant cardiac tumors can present with a variety of symptoms, mainly via obstruction of blood flow, local invasion of myocardium causing arrhythmias, metastasis and pleural effusion, and embolization. The echocardiographic examination focuses on the anatomic location and extent of the tumor involvement, the physiologic consequences of the tumor (e.g., valve regurgitation, chamber obliteration, obstruction) and associated findings (pericardial effusion, evidence of tamponade physiology). ${ }^{17}$ Echocardiography usually shows a broad-based right atrial mass near the inferior vena cava. ${ }^{8}$ Although TTE is a reasonable initial screening tool, TEE may offer important clues as to the malignant nature of the lesion by 
showing intramyocardial and, for right atrial masses, vena caval invasion. ${ }^{5}$ The superior soft tissue characterization possible with CT and especially MRI also allows for the determination of the degree of tumor infiltration..$^{5}$ Sarcomas are usually aggressive tumors that proliferate rapidly and have a poor prognosis. 5 Death is due to widespread infiltration of the myocardium, obstruction of flow within the heart or distant metastases and most often occurs from a few weeks to 2 years after the onset of symptoms with the median survival being 6 to 12 months. $^{5}$

\section{Angiosarcomas}

Angiosarcomas are the most common primary malignant cardiac tumors accounting for $30 \%$ of primary cardiac sarcomas. ${ }^{5}$ They commonly occur in middle aged men. ${ }^{24}$ These tumors have a striking predilection for the right atrium and may be either intracavitary and polypoid or diffuse and infiltrative. ${ }^{5}$ Angiosarcomas have a nonspecific clinical course and physical examination, and are usually diagnosed postmortem. ${ }^{25}$ Although clinical presentation is often nonspecific, recurrent unexplained pericardial effusion is a common manifestation and should raise a high index of suspicion. ${ }^{24}$ However, even if diagnosis is made promptly, the majority of patients already have metastasis at the time of discovery. The prognosis of cardiac angiosarcoma is notoriously grave, with a median survival time of 3 to 6 months. ${ }^{24}$

\section{Rhabdomyosarcomas}

Embryonal rhabdomyosarcomas account for $10 \%$ of all primary cardiac sarcomas, but are the most common cardiac malignancy in infants and children. ${ }^{5}$ They usually arise from the ventricular walls and frequently interfere with valvular motion because of their intracavitary bulk. ${ }^{7}$ The right atrium is involved most often, where the appearance can resemble myxoma. ${ }^{3}$ Other less common malignant tumors consist of various sarcomas: myxosarcoma, liposarcoma, fibrosarcoma, leiomyosarcoma, osteosarcoma, synovial sarcoma, undifferentiated sarcoma, reticulum cell sarcoma, neurofibrosarcoma and malignant fibrous histiocytoma. ${ }^{26}$

\section{Primary cardiac lymphoma}

Lymphomas account for 5\% of primary malignant cardiac tumors. 5 Overall, however, they are extremely rare and represent only $1 \%$ of all (benign and malignant) primary cardiac tumors. ${ }^{27}$ Primary cardiac lymphoma is defined as an extranodal non-Hodgkin's lymphoma involving only the heart and pericardium at the time of diagnosis. ${ }^{27}$ Lymphomatous involvement of the heart and/or pericardium usually occurs by dissemination, and cardiac involvement as the initial presenting manifestation of malignant non-Hodgkin's lymphoma is extremely rare. Nevertheless, its frequency has been increasing in the past 10 years, mainly in immunocompromised patients. ${ }^{27}$ Clinical presentation of primary cardiac lymphoma varies, is nonspecific and depends on which cardiac structures are involved. Patients may present with congestive heart failure, arrhythmias, pericardial effusion or superior vena cava syndrome. ${ }^{27}$ Diagnostic techniques such as electrocardiography, echocardiography, CT and MRI are nonspecific for lymphoma and confirmation of the diagnosis requires morphological examination of the tissue infiltrated by the tumor. A method with high diagnostic accuracy is necessary to ensure successful therapy for primary heart lymphoma. ${ }^{27}$ Since cytological examination of pericardial fluid is not always sensitive for malignancies, especially for lymphoma, and not all patients are compromised by the pericardial effusion, a transvenous biopsy of the tumor is preferred. ${ }^{27}$ Transvenous biopsy has been used successfully to diagnose cardiac lymphomas and is well established for obtaining adequate diagnostic tissue particles. $^{27}$

The prognosis of primary heart lymphoma is poor due to diagnostic delay and advanced stage of organ infiltration. ${ }^{27}$ The survival is generally less than a month without treatment, but has been prolonged to up to 5 years with chemotherapy and radiation therapy in selected cases. ${ }^{28}$

\section{Metastatic Tumors of the Heart}

Nonprimary (metastatic) cardiac tumors are about 20 times more common than primary cardiac tumors. ${ }^{17}$ In an autopsy series of patients with a malignancy, cardiac involvement is present in approximately $10 \%$ of cases, although clinical recognition of cardiac involvement occurs less frequently. ${ }^{17}$ Metastatic tumors can affect the heart by direct invasion of nearby malignancies such as the lung and breast, by lymphatic spread or by metastasis of distant malignancies such as lymphoma and melanoma. Metastatic spread from malignancies to the heart occurs most commonly in association with lung and breast carcinoma, lymphoma, leukemia and malignant melanoma. ${ }^{27}$ Malignant melanoma has the highest rate of cardiac metastases by any tumor, and cardiac involvement is diagnosed more now because of increasing incidence and survival of patients with melanoma. ${ }^{29}$ Melanoma has a high propensity for metastasizing to the pericardium and/or myocardium, involving the heart in more than $50 \%$ of cases. ${ }^{1}$ Metastatic disease affects the pericardium more frequently than the heart itself; consequently, pericardial effusion is the most common echocardiographic finding in patients with metastatic cardiac disease. ${ }^{3}$ Approximately $75 \%$ of metastatic cardiac diseases involve the pericardium and epicardium, presenting as a pericardial effusion. ${ }^{17}$ Therefore, pericardial effusion in a patient with known malignancy should alert the echocardiographer to the possibility of cardiac involvement. Although less common, metastatic lesions can occur in the myocardium, particularly in lymphoma and melanoma. A specific type of cardiac involvement by tumor that should be recognized by the echocardiographer is extension of renal cell carcinoma up the inferior vena cava. ${ }^{17}$ The tumor can protrude into the right atrium from the inferior vena cava as a finger-like projection. ${ }^{17}$ Identification of this tumor is important because curative en bloc resection may be possible. Common sources of metastatic cardiac tumor in the pediatric population includes lymphoma, Wilms' tumor, malignant teratoma, neuroblastoma and pleuropulmonary blastoma. ${ }^{30}$ 


\section{Intracardiac Thrombi}

Thrombi can be misinterpreted as a cardiac tumor. The presence of atrial fibrillation, enlarged atrial chamber, low cardiac output state are all features that favor the mass in question being a thrombus. ${ }^{31}$ Thrombi occur in areas of blood stasis. Left ventricular thrombi occur commonly at the apex (figures 4A and 4B) and are predominant in patients with ventricular aneurysms or patients within the first week of an ST elevation myocardial infarction (with subsequent akinetic/dyskinetic walls). Left atrial thrombi (figures 4C and 4D) are usually found in the appendage and associated with atrial fibrillation, atrial flutter, mitral stenosis or prosthetic mitral valve. TTE is the clinical procedure of choice for identification of left ventricular thrombi. ${ }^{17}$ TTE has greater than $90 \%$ sensitivity and greater than $85 \%$ specificity for detection of left ventricular thrombi and is probably superior to the sensitivity and specificity of TEE, especially for apical thrombi. ${ }^{32}$ TEE is rarely helpful and may be less sensitive, as the apex may not be visualized in standard image planes and the left ventricular apex is at considerable distance from the tranducer, thereby limiting resolution of structural detail. ${ }^{17}$ However, in patients that TTE is limited secondary to chronic obstructive pulmonary disease or obesity, TEE may be required. On the other hand, atrial thrombi frequently require TEE for diagnosis, with the appendage best seen at 30 to 40 degrees of rotation from the basilar short-axis view. ${ }^{32}$ TEE imaging has a much greater sensitivity and a high negative predictive value for the diagnosis of left atrial thrombi because the left atrium lies closest to the transducer, allowing the left atrial appendage to be viewed clearly. Thrombi in the atria and atrial appendages are more commonly identified by TEE. These thrombi, usually located in the appendages, are most commonly identified in the setting of atrial fibrillation or mitral valve disease. ${ }^{14}$

Formation of thrombi in the right heart is rare. Most thrombi in the right heart are venous thrombi that embolized to the heart. TEE is better able to resolve the presence, extent and attachment of right-sided heart thrombi. ${ }^{17}$

Compared to other diagnostic procedures, MRI and CT offer a similar sensitivity of about $90 \%$, with a slightly better specificity as compared to $2 \mathrm{DE} .{ }^{12}$ Spontaneous echo contrast (SEC), or "smoke," is the swirling, hazy echocardiographic appearance associated with low blood flow. ${ }^{1}$ Several investigators consider SEC to be a pre-thrombotic condition, although it is not clearly established that SEC actually leads to thrombus formation. ${ }^{1}$ Regardless of the cause and effect, the presence of SEC has been consistently associated with increased risk of thromboembolism. ${ }^{1}$

\section{Clinical Value of Common Imaging Modalities Utilized for Evaluation of Cardiac Masses}

Echocardiography allows dynamic evaluation of intracardiac masses with the advantage, compared to other tomographic techniques, that both the anatomic extent and the physiologic consequences of the mass can be evaluated. ${ }^{17}$ This is important because although $75 \%$ of primary cardiac tumors are benign, even a benign tumor can have significant physiologic consequences secondary to compression or obstruction of blood flow.

Although echocardiography has many advantages for evaluating cardiac tumors, there are some limitations. Bone and lung interference remains a major limitation of echocardiography and renders this real-time imaging technique suboptimal in patients with chronic obstructive pulmonary disease or narrow rib spaces. ${ }^{33}$ Aside from suboptimal image quality in some patients, echocardiography has limited field of view compared to MRI and CT (discussed later). TEE can provide further information when limited acoustic viewing restricts optimal evaluation. In one study of 17 patients investigated for cardiac tumor, TTE showed 4 false-positives and 2 false-negatives, whereas TEE resulted in only 1 false-positive and no false-negatives. ${ }^{3}$ Thus, TEE has superior specificity and sensitivity for diagnosing cardiac tumors. ${ }^{3}$ The potential advantages of TEE include improved resolution of the tumor and its attachment, the ability to detect some masses not visualized by TTE and improved visualization of right atrial tumors. ${ }^{5}$ Both TEE and TTE are equally effective in visualizing tumors originating from the heart. However, when comparing TTE with TEE in patients with mediastinal tumors, marked differences were seen. ${ }^{34}$ Tumors originating from the mediastinum were 2.9 times less likely to be detected by TTE than TEE, and TEE was also superior in diagnosing tumor infiltration and invasion of the heart and the great vessels. ${ }^{34}$ In patients with malignant tumors originating in the heart, TEE provided superior information to the TTE approach regarding the site of attachment, intra- and extracardiac expansion and the morphological characterization of the tumor surface. ${ }^{34}$ The sensitivity of these criteria for identifying patients with histologically proven malignant tumors was only $8 \%$ when the transthoracic approach was used, but increased to $40 \%$ using transesophageal imaging. ${ }^{34}$ In conclusion, although TEE appears to be superior to TTE in many patients, it is not warranted on a routine basis and should be considered when the transthoracic study is suboptimal or where TTE is impossible (e.g., during surgery). 5,35

Echocardiographic contrast perfusion imaging has been used to characterize vascularity of cardiac masses and differentiate malignant/highly vascular tumors from benign tumors and thrombi. Since most benign cardiac tumors are relatively avascular, the vascularity of a mass may be a feature that can help differentiate benign from malignant tumors. ${ }^{36}$ In the study by Kirkpatrick et al $^{37}$ echocardiographic contrast perfusion imaging showed that compared with the adjacent myocardium, malignant and vascular tumors hyper-enhance, whereas stromal tumors and thrombi hypo-enhance. Malignant or highly vascular tumors demonstrated greater enhancement than the myocardium, myxomas (which have a poor blood supply) demonstrated less perfusion than myocardium and thrombi (generally avascular) demonstrated no perfusion. ${ }^{37}$ In the past, CT scans could not define intracardiac anatomy 
because of long scan times relative to cardiac and respiratory motion. Recent advances in CT have greatly improved its application to cardiac imaging. Of note, data acquisition is sufficiently rapid (50 to $100 \mathrm{msec}$ ) for electron beam tomography (EBT) that cardiac gating is not absolutely necessary, permitting adequate imaging in patients with irregular rhythms. ${ }^{5} \mathrm{CT}$ provides a high degree of soft tissue discrimination, which is helpful in defining the degree of myocardial infiltration. ${ }^{5}$ It appears to be useful to determine the degree of myocardial invasion and involvement of extracardiac structures in patients with suspected malignancy of the heart because it allows evaluation of extracardiac structures. Mousseaux et $\mathrm{al}^{38}$ demonstrated that EBT provides additional anatomical information over cross-sectional echocardiography in all patients with malignant tumors and it provides better delineation of the relation between intracavity masses and normal structures, including the mural site of attachment and tumor extensions. On the other hand, EBT provides no additional information over cross-sectional echocardiography in patients with myxomas, thus cross-sectional echocardiography alone is sufficient to establish the need for surgery. ${ }^{38}$ In $70 \%$ of the cases studied by Mousseaux et al ${ }^{38}$ EBT modified the diagnosis or confirmed a diagnosis that had been questionable by cross-sectional echocardiography. Gross et $\mathrm{al}^{39}$ demonstrated that CT evaluates extent of disease, including invasion of contiguous vessels and pulmonary metastases, better than echocardiography. Furthermore, CT was able to directly image neoplastic pulmonary emboli. ${ }^{39} \mathrm{CT}$ can also be used to assess for calcification, which may be useful in the diagnosis of rhabdomyomas, fibromas, teratomas, myxomas, hemangiomas and osteosarcomas. ${ }^{5}$ Although CT has some advantages over echocardiography, it is more invasive because it requires the use of ionization radiation and frequently an intravascular injection of contrast medium. ${ }^{33}$ This limits the use of contrast CT in patients with compromised renal function.

MRI is also useful in evaluation of cardiac masses. MRI can be used to verify intracardiac masses found on $2 \mathrm{DE}$ and to exclude a mass when the echocardiogram is equivocal. ${ }^{40} \mathrm{MRI}$ can provide information about tissue composition of cardiac masses when images are obtained with pulse sequences of different T1 and T2 weightings. ${ }^{41}$ T1-weighted and T2-weighted dual-inversion recovery fast spin-echo sequences offer detailed morphological information, with T1-weighted images providing excellent soft tissue characterization and T2-weighted images providing superior tissue contrast and demonstration of fluid components. 5

MRI allows this tissue characterization by comparing the T1 and T2 values of the masses to a reference tissue, which is fat. ${ }^{12}$ Tissue characterization by MRI is limited to the diagnosis of myxomas, fibromas, thrombi and fatty tissue. ${ }^{12}$ The signal features of malignant tumors are ambivalent and do not permit a tissue diagnosis. ${ }^{12}$ The primary contribution of MRI is not the ability to obtain tissue diagnosis, but rather its ability to delineate the anatomic extent and to aid in treatment planning. ${ }^{42}$ In addition to having a large field of view that allows imaging of the paracardiac space and the great vessels, MRI enables high-resolution tomography in three dimensions, is nonionizing and generates intravascular and soft tissue contrast without the need for contrast medium. ${ }^{33,43}$ Amparo et al ${ }^{44}$ demonstrated that MRI could provide as useful information as echocardiography, CT and angiography combined in the evaluation of cardiac and paracardiac masses. However, considering the expense, it is not cost effective. Furthermore, unlike echocardiography, MRI cannot be brought to the bedside. Additionally, use of MRI is contraindicated in patients with cardiac pacemakers, implanted defibrillators and other similar mechanical devices.

Both CT with MRI allow imaging and evaluation of extracardiac structures. Comparing CT and MRI, CT is faster and easier to perform, while MRI provides better soft-tissue contrast and allows much greater flexibility in the selection of imaging planes. ${ }^{11}$ Another difference is that calcium cannot be identified by MRI and when calcification of a mass is suspected, CT offers a distinct advantage over the other modalities. ${ }^{11,33}$

\section{Conclusion}

The diagnosis of intracardiac tumors has improved enormously in recent years. Echocardiography has evolved since its inception in the mid-1950s and has now become the screening tool of choice for cardiac tumors. ${ }^{5}$ Two-dimensional echocardiography is the primary diagnostic imaging modality for the evaluation of cardiac masses. ${ }^{6}$ The judicious use of both echocardiography and CT or MRI may be needed in an individual patient for optimal clinical decision making. ${ }^{45}$ Each technique has inherent advantages as well as limitations, including expense, radiation exposure, image acquisition time and lack of portability. ${ }^{14}$ However, $2 \mathrm{DE}$ is a non-invasive, cost-effective imaging modality that offers real-time, high spatial and temporal resolution imaging for initial evaluation of intracardiac masses.

Three-dimensional echocardiography is a useful tool that may provide additional information or better visualization of intracardiac masses. ${ }^{46,47}$ It is conceivable that echocardiography will continue to evolve and play a prominent role in clinical medicine and that improvements in technology will likely provide better images and diagnostic information in the future.

\section{References}

1. Masses, tumors, and source of embolus. In Feigenbaum $H$, Armstrong WF, Ryan T. Feigenbaum's echocardiography. $6^{\text {th }}$ ed. Philadelphia, PA: Lippincott Williams \& Wilkins; 2005. 701-733.

2. Dittrich HC. Clinical transesophageal echocardiography. St. Louis, MO: Mosby Year Book; 1992. 85.

3. Goldman JH, Foster E. Transesophageal echocardiographic (TEE) evaluation of intracardiac and pericardial masses. Cardiol Clin 2000;18:849-860. 
4. Nadra I, Dawson D, Schmitz SA, Punjabi PP, Nihoyannopoulos P. Lipomatous hypertrophy of the interatrial septum: a commonly misdiagnosed mass often leading to unnecessary cardiac surgery. Heart 2004;90:e66.

5. Braunwald E. Heart disease: A textbook of cardiovascular medicine. $6^{\text {th }}$ ed. Philadelphia, PA: WB Saunders Co; 2001.

6. Meng Q, Lai H, Lima J, Tong W, Qian Y, Lai S. Echocardiographic and pathologic characteristics of primary cardiac tumors: a study of 149 cases. Int J Cardiol 2002;84:69-75.

7. Castorino F, Masiello P, Quattrocchi E, Di Benedetto G. Primary cardiac rhabdomyosarcoma of the left atrium: an unusual presentation. Tex Heart Inst J 2000;27:206-208.

8. Shapiro LM. Cardiac tumours: diagnosis and management. Heart 2001;85:218-222.

9. Endo A, Ohtahara A, Kinugawa T, Mori M, Fujimoto Y, Yoshida A, Kuroda H, Mori T, Mashiba H, Shigemasa C. Characteristics of 161 patients with cardiac tumors diagnosed during 1993 and 1994 in Japan. Am J Cardiol 1997;79:1708-1711.

10. Yuehua L, Jing G, Kai F, Hongwei W, Jingjing L. Left atrial myxoma presenting with erythematous macules and loss of memory. Clin Exp Dermatol 2003;28:383-386.

11. Araoz PA, Mulvagh SL, Tazelaar HD, Julsrud PR, Breen JF. CT and MR imaging of benign primary cardiac neoplasms with echocardiographic correlation. Radiographics 2000;20:1303-1319.

12. Hoffmann U, Globits S, Frank H. Cardiac and paracardiac masses. Current opinion on diagnostic evaluation by magnetic resonance imaging. Eur Heart J 1998;19:553-563.

13. Vidaillet HJ Jr, Seward JB, Fyke FE 3rd, Su WP, Tajik AJ. "Syndrome myxoma": a subset of patients with cardiac myxoma associated with pigmented skin lesions and peripheral and endocrine neoplasms. Br Heart J 1987;57:247255.

14. Lobo A, Lewis JF, Conti CR. Intracardiac masses detected by echocardiography: case presentations and review of the literature. Clin Cardiol 2000;23:702-708.

15. Kindman LA, Wright A, Tye T, Seale W, Appleton C. Lipomatous hypertrophy of the interatrial septum: characterization by transesophageal and transthoracic echocardiography, magnetic resonance imaging, and computed tomography. J Am Soc Echocardiogr 1988;1:450-454.

16. Sun JP, Asher CR, Yang XS, Cheng GG, Scalia GM, Massed AG, Griffin BP, Ratliff NB, Stewart WJ, Thomas JD. Clinical and echocardiographic characteristics of papillary fibroelastomas: a retrospective and prospective study in 162 patients. Circulation 2001;103:2687-2693.

17. Otto CM. Textbook of clinical echocardiography. $2^{\text {nd }}$ ed. Philadelphia, PA: WB Saunders Company; 2000. 350-370.

18. Krasuski RA, Hesselson AB, Landolfo KP, Ellington KJ, Bashore TM. Cardiac rhabdomyoma in an adult patient presenting with ventricular arrhythmia. Chest 2000;118:12171221.

19. Di Liang C, Ko SF, Huang SC. Echocardiographic evaluation of cardiac rhabdomyoma in infants and children. J Clin Ultrasound 2000;28:381-386.

20. Wu SS, Collins MH, de Chadarevian JP. Study of the regression process in cardiac rhabdomyomas. Pediatr Dev Pathol 2002;5:29-36.

21. Cotton JL, Kavey RE, Palmier CE, Tunnessen WW Jr. Cardiac tumors and the nevoid basal cell carcinoma syndrome. Pediatrics 1991;87:725-728.

22. Kimonis VE, Mehta SG, Digiovanna JJ, Bale SJ, Pastakia B. Radiological features in 82 patients with nevoid basal cell carcinoma (NBCC or Gorlin) syndrome. Genet Med 2004;6:495-502.

23. Araoz PA, Eklund HE, Welch TJ, Breen JF. CT and MR imaging of primary cardiac malignancies. Radiographics 1999; 19:1421-1434.
24. Lee CH, Chan GS, Chan WM. Unexplained recurrent pericardial effusion: a lethal warning? Heart 2003;89:e11.

25. Komamura K, Miyatake K. Transthoracic Doppler flow images detect cardiac angiosarcoma earlier than other imaging modalities. Heart 2002;87:28.

26. Vander Salm TJ. Unusual primary tumors of the heart. Semin Thorac Cardiovasc Surg 2000;12:89-100.

27. Daus H, Bay W, Harig S, Schneider G, Feiden W, Schieffer H. Primary lymphoma of the heart: report of a case with histological diagnosis of the transvenously biopsied intracardiac tumor. Ann Hematol 1998;77:139-141.

28. Gowda RM, Khan IA. Clinical perspectives of primary cardiac lymphoma. Angiology 2003;54:599-604.

29. Gibbs P, Cebon JS, Calafiore P, Robinson WA. Cardiac metastases from malignant melanoma. Cancer 1999;85:78-84.

30. Huh J, Noh CI, Kim YW, Choi JY, Yun YS, Shin HY, Ahn HS, Kim YJ. Secondary cardiac tumor in children. Pediatr Cardiol 1999;20:400-403.

31. Alam M. Pitfalls in the echocardiographic diagnosis of intracardiac and extracardiac masses. Echocardiography 1993;10:181-191.

32. Roldan CA. The ultimate echo guide. Philadelphia, PA: Lippincott Williams \& Wilkins; 2005. 189-203.

33. Freedberg RS, Kronzon I, Rumancik WM, Liebeskind D. The contribution of magnetic resonance imaging to the evaluation of intracardiac tumors diagnosed by echocardiography. Circulation 1988;77:96-103.

34. Geibel A, Kasper W, Keck A, Hofmann T, Konstantinides S, Just H. Diagnosis, localization and evaluation of malignancy of heart and mediastinal tumors by conventional and transesophageal echocardiography. Acta Cardiol 1996;51:395-408.

35. Hanrath P. Imaging techniques: Transoesophageal echo-Doppler in cardiology. Heart 2001;86:586-592.

36. Lepper W, Shivalkar B, Rinkevich D, Belcik T, Wei K. Assessment of the vascularity of a left ventricular mass using myocardial contrast echocardiography. J Am Soc Echocardiogr 2002;15:1419-1422.

37. Kirkpatrick JN, Wong T, Bednarz JE, Spencer KT, Sugeng L, Ward RP, DeCara JM, Weinert L, Krausz T, Lang RM. Differential diagnosis of cardiac masses using contrast echocardiographic perfusion imaging. J Am Coll Cardiol 2004;43:1412-1419.

38. Mousseaux E, Hernigou A, Azencot M, Sapoval M, Auguste M, Gaux JC. Evaluation by electron beam computed tomography of intracardiac masses suspected by transesophageal echocardiography. Heart 1996;76:256-263.

39. Gross BH, Glazer GM, Francis IR. CT of intracardiac and intrapericardial masses. AJR Am J Roentgenol 1983;140:903-907.

40. Winkler M, Higgins CB. Suspected intracardiac masses: evaluation with MR imaging. Radiology 1987;165:117-122.

41. Tuna IC, Julsrud PR, Click RL, Tazelaar HD, Bresnahan DR, Danielson GK. Tissue characterization of an unusual right atrial mass by magnetic resonance imaging. Mayo Clin Proc 1991;66:498-501.

42. Lund JT, Ehman RL, Julsrud PR, Sinak LJ, Tajik AJ. Cardiac masses: assessment by MR imaging. AJR Am J Roentgenol 1989;152:469-473.

43. Hoffmann U, Globits S, Schima W, Loewe C, Puig S, Oberhuber G, Frank H. Usefulness of magnetic resonance imaging of cardiac and paracardiac masses. Am J Cardiol 2003;92:890-895.

44. Amparo EG, Higgins CB, Farmer D, Gamsu G, McNamara M. Gated MRI of cardiac and paracardiac masses: initial experience. AJR Am J Roentgenol 1984;143:1151-1156.

45. Menegus MA, Greenberg MA, Spindola-Franco H, Fayemi A. Magnetic resonance imaging of suspected atrial tumors. Am Heart J 1992;123:1260-1268. 
46. Ahmed S, Nanda NC, Miller AP, Nekkanti R, Yousif AM, Pacifico AD, Kirklin JK, McGiffin DC. Volume quantification of intracardiac mass lesions by transesophageal three-dimensional echocardiography. Ultrasound Med Biol 2002;28:1389-1393.

47. Lokhandwala J, Liu Z, Jundi M, Loyd A, Strong M, Vannan M. Three-dimensional echocardiography of intracardiac masses. Echocardiography 2004;21:159-163.

\section{Author Affiliations}

Moluk Mirrasouli Ragland, DO, Department of Medicine, University of North Texas Health Science Campus, 855

Montgomery Road, 4th Floor, Fort Worth, Texas 76107

Tahir Tak, MD, PhD, Department of Medicine, University of North Texas Health Science Campus, 855 Montgomery

Road, 4th Floor, Fort Worth, Texas 76107 